\title{
Isoflavones Suppress Cyp26b1 Expression in the Murine Colonic Lamina Propria
}

\author{
Takeshi Yamamoto, ${ }^{*, a, \#}$ Yuka Nagata, ${ }^{a, b, \#}$ Shusaku Hayashi, ${ }^{a}$ and Makoto Kadowaki ${ }^{a}$ \\ ${ }^{a}$ Division of Gastrointestinal Pathophysiology, Institute of Natural Medicine, University of Toyama; 2630 Sugitani, \\ Toyama 930-0194, Japan: and ${ }^{b}$ Faculty of Pharmaceutical Sciences, Institute of Medical, Pharmaceutical and Health \\ Sciences, Kanazawa University; Kakuma-machi, Kanazawa, Ishikawa 920-1192, Japan. \\ Received April 17, 2020; accepted September 5, 2020
}

Isoflavones have many biological activities and are major bioactive components of kakkonto, a traditional Japanese herbal medicine. We previously reported that the combined therapy of oral immune therapy (OIT) and kakkonto downregulates the mRNA expression of Cyp26b1, a major retinoic acid (RA)-degrading enzyme, in the colon of food allergy mice and thereby ameliorates allergic symptoms. In this study, we evaluated the effects of various isoflavones on Cyp26b1 expression in primary cultured lamina propria (LP) cells isolated from the mouse colon. The mRNA expression of Cyp26b1 was extremely downregulated by all isoflavones tested in the LP cells except for puerarin. In particular, genistein and genistin markedly suppressed Cyp26b1 mRNA expression without affecting RA-synthesizing enzyme expression. Moreover, to evaluate the effects of isoflavones on allergic reactions, genistein and genistin were administered to ovalbumin (OVA)induced food allergy mice. Oral administration of genistin suppressed the development of allergic symptoms. These results raise the possibility that isoflavones elevated the level of RA in the colon by inhibiting RA degradation and then the high concentration of $\mathrm{RA}$ in the colon might exert immunosuppressive and antiallergic effects on food allergy mice.

Key words genistin; genistein; colonic lamina propria; Cyp26b1; food allergy

\section{INTRODUCTION}

Cyp26 has been characterized as a pivotal enzyme responsible for retinoic acid (RA) degradation. ${ }^{1)}$ Cyp26 controls RA homeostasis in concert with aldehyde dehydrogenase (Aldh), an RA-synthesizing enzyme, to regulate the tissue level of RA. In the intestine, Cyp26b1 appears to be expressed in $\mathrm{CD}^{+} \mathrm{T}$ cells and $\mathrm{CD}^{+} \mathrm{T}$ cells in the gut-associated lymphoid tissue, whereas Aldh1al is highly expressed in epithelial cells. ${ }^{2-4)} \mathrm{RA}$ is the active derivative of vitamin $\mathrm{A}$ and has been implicated in the regulation of intestinal mucosal immune responses. In particular, RA-induced regulatory $\mathrm{T}$ (Treg) cells and tolerogenic $\mathrm{CD}_{103}{ }^{+}$dendritic cells (DCs) exert suppressive functions to prevent excessive immune responses. ${ }^{5}$

Food allergy is considered to result from a disruption of the intestinal mucosal immune system. We have reported that kakkonto, a traditional Japanese herbal medicine (Kampo medicine), significantly suppresses the occurrence of ovalbumin (OVA)-induced allergic diarrhea in food allergy mice and kakkonto significantly induces $\mathrm{Foxp} 3^{+} \mathrm{CD} 4^{+}$Tregs in the colon of food allergy mice as a novel mechanism underlying the therapeutic action. ${ }^{6,7)}$ Subsequently, we have demonstrated that compared with oral immune therapy (OIT), the combined therapy of OIT and kakkonto significantly further alleviates the allergic symptoms in food allergy mice and markedly downregulates the mRNA expression of Cyp26b1 in the proximal colon of food allergy mice, ${ }^{8)}$ suggesting that some components of kakkonto attenuate Cyp26b1 function in the colon and exert anti-allergic and anti-inflammatory effects. Kudzu root, a constituent herb in kakkonto, is traditionally used

\footnotetext{
${ }^{\#}$ These authors contributed equally to this work.

*To whom correspondence should be addressed. e-mail: ty@inm.u-toyama.ac.jp
}

in Japan and China as a source of starch and contains large amounts of various isoflavones, such as daidzin, puerarin, daidzein and genistein. ${ }^{9-11)}$ Many of the biological activities of kudzu root are attributed to these isoflavones. ${ }^{12-14)}$ In addition, it is shown that genistein, one of isoflavones, has an inhibitory effect of Cyp26b1 expression in a human hepatoblastoma cell line ${ }^{15)}$ whereas puerarin, an isoflavone derivative, significantly enhances the mRNA expression of Aldh1al in colonic epithelial cells in food allergy mice and thereby attenuates allergic symptoms in these mice. ${ }^{16)}$ Thus, using primary cultured colonic lamina propria (LP) cells isolated from the mouse colon, we examined the effects of isoflavones on Cyp26b1 mRNA expression. This study provides evidences for the potential roles of isoflavones in modulating RA metabolism.

\section{MATERIALS AND METHODS}

Mice BALB/c mice were purchased from Japan SLC (Japan). All mice were housed in a specific pathogen-free environment. All animal experiments were approved by the Animal Experiment Committee of the University of Toyama (Approval Nos.: A2018 INM-4 and A2015 INM-3).

Drugs Kakkonto (product code: TJ-1, Lot. No. 2160001010) and a dried extract powder of kudzu root (Puerariae radix) (Lot. No. 2181021010) were purchased as the spray-dried product form Tsumura \& Co. (Tokyo, Japan). Kakkonto is composed of 7 species of medicinal plants: Puerariae radix, Ephedrae herba, Zizyphi fructus, Paeoniae radix, Cinnamomi cortex, Zingiberis rhizoma and Glycyrrhizae radix $(22.2,16.7$, 16.7, 11.1, 11.1, 11.1 and 11.1\%). These medicinal plants are authenticated by identification according to the methods of the Japanese Pharmacopoeia and the standards of Tsumura \& Co. 
The medicinal herbs were extracted with boiling water and the extract solution was separated from the insoluble matter. The extract solution was spray-dried to obtain a dried extract powder. A three-dimensional HPLC profiles of kakkonto and an extract of kudzu root are shown in Supplementary Figs. 1, 2.

Cell Culture Mononuclear cells were isolated from the spleen (SP), mesenteric lymph node (MLN) and intestinal LP of mice with OVA-induced allergic diarrhea. Food allergy induction and LP cell isolation were performed as previously described. ${ }^{6,7)}$ Briefly, mice were sensitized and challenged with OVA to induce food allergy-related diarrhea. The excised colon was treated with ethylenediaminetetraacetic acid (EDTA) to remove the epithelial cells and digested with collagenase. Isolated LP cells were purified by centrifugation in a Percoll solution. Kakkonto and a dried extract powder of kudzu root were dissolved in distilled water. Isoflavones were dissolved in dimethyl sulfoxide (DMSO) and assayed at concentrations ranging from 1 to $100 \mu \mathrm{M}$. These test drugs were applied to cultured LP cells for $24 \mathrm{~h}$.

Quantitative PCR Cells were lysed in Sepasol RNA I Super, and total RNA was isolated following the manufacturer's instructions (Nacalai Tesque, Japan). cDNA was synthesized using an RT reagent kit (TaKaRa Bio, Japan). Transcription levels were measured by real-time PCR using TB Green Premix and a TaKaRa TP800 system (TaKaRa Bio) and normalized to the mRNA levels of glyceraldehyde3-phosphate dehydrogenase (GAPDH) which was used as an internal control for each sample. Primer sequences were reported previously. $^{8)}$

In Vivo Study The murine food allergy model was performed as described above. Isoflavones were suspended in a methylcellulose solution and administered to mice orally $1 \mathrm{~h}$ before oral challenge with OVA during the elicitation phase of the allergic symptoms. Allergic symptoms (diarrhea) were assessed by visually monitoring the mice for up to $1 \mathrm{~h}$ following oral OVA challenge.7)

Statistical Analysis Statistical differences were determined by Student's $t$-test (between 2 groups), one-way ANOVA followed by Dunnett's multiple-comparison test (among multiple groups) or the chi-square test.

\section{RESULTS}

Kudzu Root Downregulates Cyp26b1 mRNA Expression in LP Cells The mRNA expression level of Cyp26b1 in the LP was much higher than that in other lymphoid tissues (Fig. 1A). We previously reported that kakkonto administration suppressed the mRNA expression of Cyp26b1 in the colon of OVA-induced food allergy mice. $\left.{ }^{6}\right)$ Based on this previous evidence, we assessed the direct effects of kakkonto on LP cells isolated from the colon of OVA-induced food allergy mice. Kakkonto treatment $(500 \mu \mathrm{g} / \mathrm{mL})$ significantly suppressed Cyp26b1 mRNA expression in the LP cells (Fig. 1B), and the inhibitory effect was dose dependent (data not shown). However, kakkonto treatment $(500 \mu \mathrm{g} / \mathrm{mL})$ produced no change in the transcription of Aldhla1, an RA-synthesizing enzyme (Fig. 1B). To identify the effective components of kakkonto that downregulated Cyp26b1 mRNA expression, each extract was directly applied to LP cells isolated from the colon of OVA-induced food allergy mice. Kudzu root $(500 \mu \mathrm{g} / \mathrm{mL})$, the main and characteristic medicinal herb in kakkonto, significantly suppressed the transcription of Cyp26b1 (Fig. 1C), and the inhibitory effect was dose dependent (data not shown), indicating that the effect of kakkonto on colonic Cyp26b1 regulation is mostly attributed to kudzu root. However, kudzu root also suppressed the transcription of Aldhla1.

Isoflavones Downregulate Cyp26b1 mRNA Expression in LP Cells Kudzu root contains multiple isoflavones, such as daidzin, puerarin, daidzein and genistein. ${ }^{7-9)}$ To evaluate the effect of isoflavones on Cyp26b1 expression, we treated LP cells isolated from the colon with different types of isoflavones $(100 \mu \mathrm{M})$. Multiple isoflavones, except for puerarin, markedly suppressed the mRNA expression of Cyp26b1 in the LP cells (Fig. 2). In particular, genistein and genistin suppressed Cyp26b1 mRNA expression without causing any changes in Aldhlal mRNA expression, while the other isoflavones tended to reduce the mRNA expression of Aldhla1. Genistein and genistin extremely suppressed Cyp26b1 mRNA expression even at $1 \mu \mathrm{M}$ (Fig. 3).

Isoflavone Suppresses Food Allergic Diarrhea in Mice To address the question of whether the reduction of Cyp26b1 mRNA expression induced by isoflavones results in
A

\section{Cyp26b1}

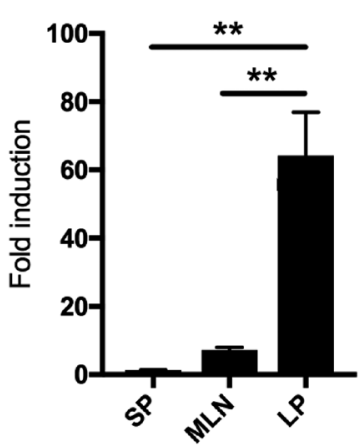

B Cyp26b1

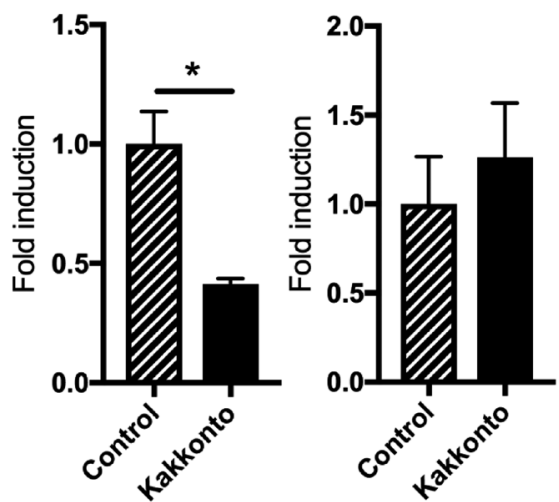

C

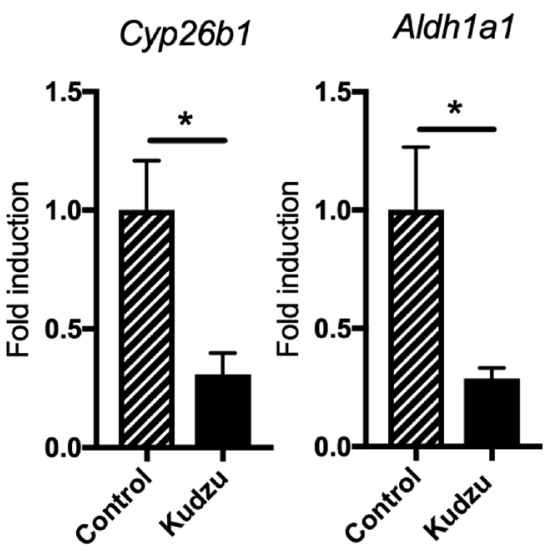

Fig. 1. Kudzu Root Downregulates Cyp26b1 mRNA Expression in LP Cells

The transcriptional levels of Cyp26b1 and Aldh1al were analyzed after $24 \mathrm{~h}$ of culture. (A) Relative gene expression was compared among mononuclear cells from the spleen (SP), mesenteric lymph node (MLN) and intestinal lamina propria (LP) $(n=3-5)$. (B, C) LP cells were treated with kakkonto (500 $\mu$ g/mL) or kudzu root extract $(500 \mu \mathrm{g} / \mathrm{mL})(n=3)$. Data are expressed as the mean \pm standard error (S.E.). ${ }^{*} p<0.05, * * p<0.01$. 
Cyp26b1

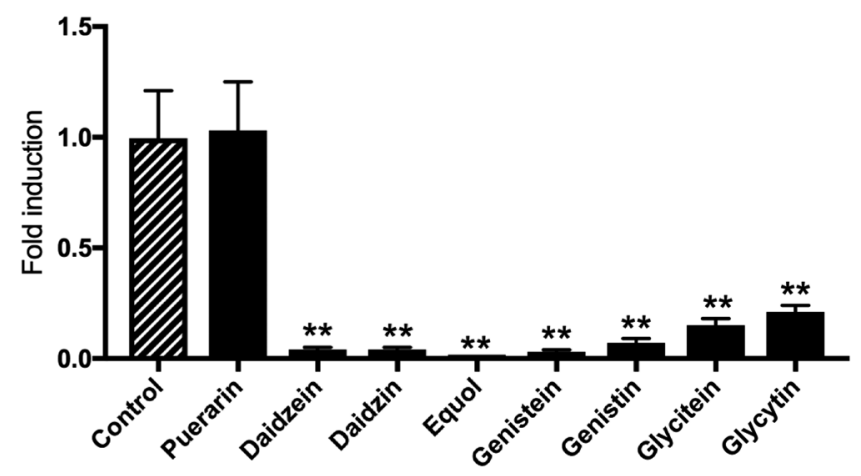

Aldh1a1

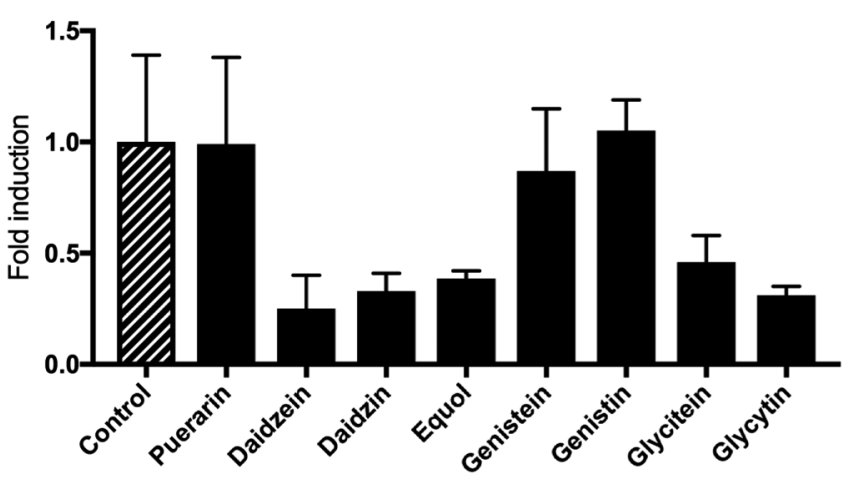

Fig. 2. Isoflavones Downregulate Cyp26b1 mRNA Expression in LP Cells Isoflavones $(100 \mu \mathrm{M})$ were used to treat LP cells. The transcriptional levels of Cyp26b1 and Aldhla1 were analyzed after $24 \mathrm{~h}$ of culture. Data are expressed as the mean \pm S.E. $(n=3-6) . * * p<0.01$ ( $v$ s. control).

\section{Cyp26b1}

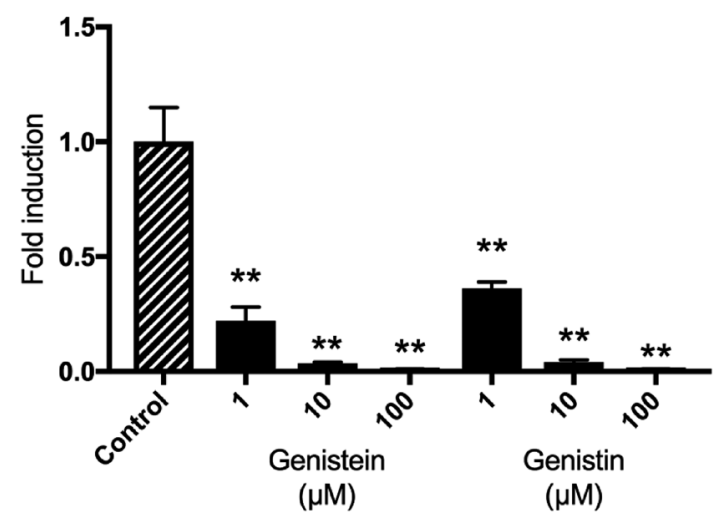

Fig. 3. Genistein and Genistin Downregulate Cyp26b1 mRNA Expression in LP Cells

Genistein and genistin were used to treat LP cells at concentrations ranging from 1 to $100 \mu \mathrm{M}$. The transcriptional levels of Cyp26b1 were analyzed after $24 \mathrm{~h}$ of culture. Data are expressed as the mean \pm S.E. $(n=3-6)$. ${ }^{*} * p<0.01$ (vs. control).

antiallergic effects, we evaluated food allergic responses in isoflavone-treated mice (Fig. 4A). Genistein and genistin were administrated to OVA-sensitized mice $1 \mathrm{~h}$ before each OVA challenge. OVA-induced development of food allergic diarrhea was significantly suppressed by genistin treatment, and most likely suppressed by genistein treatment compared to vehicle treatment (Fig. 4B).

\section{DISCUSSION}

We have demonstrated that most isoflavones clearly downregulate Cyp26b1 mRNA expression in colonic LP cells, indicating that most isoflavones have an ability to associate with the RA metabolism in the colonic mucosa through the inhibition of RA degradation. An increased RA level in the colon has been known to enhance immunosuppressive Treg cell responses. Therefore, we conclude that isoflavones suppress excessive immune responses against food antigens in the colon and thereby attenuate the development of food allergic diarrhea.

To date, the biological functions of isoflavones have been widely reported. This study identified that the isoflavones have the ability to reduce Cyp26b1 mRNA expression in the LP cells of food allergy mice. Our previous report showed that oral administration of kakkonto results in the suppression of Cyp26b1 transcription in the colon of food allergy mice and thereby attenuated food allergy-related diarrhea. ${ }^{6}$ ) Taken together, these findings indicate that the isoflavones in kudzu root extract have biological effects on RA metabolism due to the suppression of Cyp26b1 in the colon of kakkonto-treated food allergy mice.

Isoflavones extremely downregulated the mRNA expression of Cyp26b1 in the primary cultured LP cells without inducing significant changes in the mRNA expression of Aldhla1. In particular, among the isoflavones assessed in the present study, genistein and genistin were the most effective in driving the accumulation of RA in the colon of food allergy mice. We have already demonstrated that selective downregulation of intestinal Cyp26b1 expression results in tolerogenic immunosuppression for food allergy via an RA-dependent pathway. ${ }^{8)}$ In addition, a recent study has shown that the passive transfer of $\mathrm{T}$ cells isolated from Cyp26b1-deficient mice significantly attenuates disease progression in a model of $\mathrm{T}$ cell-dependent colitis. ${ }^{4}$ It also has been reported that Aldh1a1 is highly expressed in intestinal epithelial cells and that intestinal RA production is strongly dependent on Aldhlal activity. ${ }^{17)}$ These recent studies have emphasized that the balance between Cyp26b activity and Aldhla1 activity in the colon is essential to regulate the RA concentration and maintain immune homeostasis in the intestinal LP. Consequently, the selective downregulation of Cyp26b1 mRNA expression by isoflavones, especially by genistein and genistin, can contribute to the anti-inflammatory properties of isoflavones in the colon.

In this study, we have demonstrated that isoflavones downregulated Cyp26b1 mRNA expression, but the underlying mechanisms of the downregulation remains to be clarified. It is reported that genistein promotes the hypermethylation of retinoic acid receptor $\beta 2$ gene, ${ }^{18)}$ suggesting that genistein suppresses RA signaling pathway. In addition, the activation of the RA signaling pathway increases Cyp26b1 mRNA expression. ${ }^{19,20)}$ Therefore, the suppression of the RA signaling pathway might be one of the underlying mechanisms of the suppression of Cyp26b1 mRNA expression by isoflavones.

Isoflavones are major components of kakkonto and kudzu root extract, and various kinds of isoflavones are contained in kakkonto and kudzu root extract (Supplementary Figs. 1, 2). In addition, it has been reported that genistein and genistin are also included in kudzu root extract. ${ }^{21)}$ Kakkonto and 
A

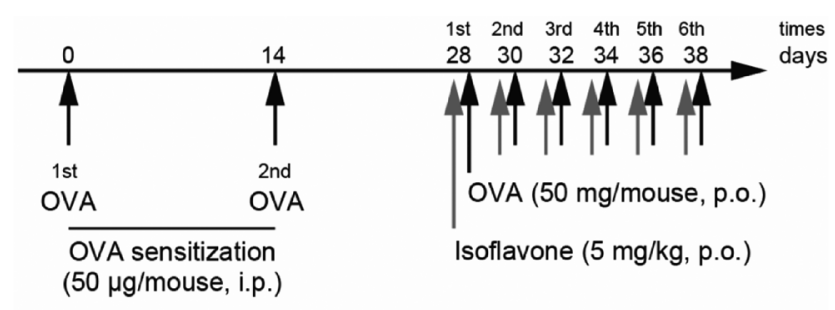

B

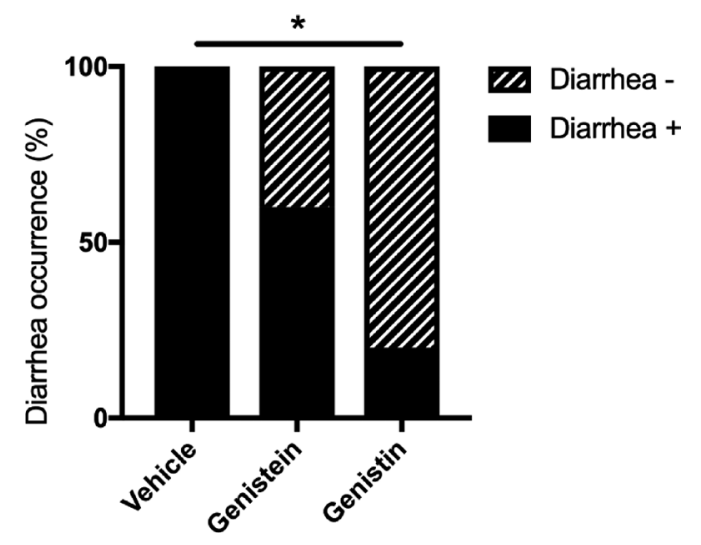

Fig. 4. Genistin Suppresses Allergic Symptoms in Food Allergy Mice

Isoflavones were administered to food allergy mice during the elicitation phase of allergic symptoms. (A) The experimental protocol is shown. (B) The occurrence of allergic diarrhea was evaluated by assessing stool conditions. Mice showing profuse liquid stool were recorded as having diarrhea. $n=10$ mice per group. $* p<0.05$.

kudzu root extract contain many components besides isoflavones (Supplementary Figs. 1, 2). Kakkonto and kudzu root extract, and isoflavones significantly suppressed mRNA expression of Cyp26b1. Thus, we think that isoflavones suppressed mRNA expression of Cyp26b1 in the LP cells through almost one mechanism without interference from many other components besides isoflavones. On the other hand, effects of kakkonto, kudzu root extract and isoflavones on mRNA expression of Aldh1al were complicated. It is reported that complex drugs composed of multiple medicinal components induce synergistic effects and additive effects. ${ }^{22}$ Therefore, we speculate that the inhibitory effect of kudzu root extract on Aldhlal mRNA expression may be caused by synergistic and/or additive effects of many components including various isoflavones contained in kudzu root extract, and that no effect of kakkonto on Aldhlal mRNA expression may be attributed to interferences by other medicinal herb extracts besides kudzu root extract.

Medical therapies using retinoids are limited due to several side effects and drug resistance. ${ }^{23)}$ In clinical studies, oral and topical RA treatments have raised safety concerns due to side effects such as teratogenicity, depression, inflammation and rash. ${ }^{23,24)}$ In addition, long-term use of RA often causes the development of resistance to the therapy due to the negative feedback mechanism involved in RA signaling. ${ }^{25)}$ To overcome these undesirable effects of RA, selective elevation of RA level in target tissues by selectively inhibiting Cyp26 enzymes is thought to be a useful remedy. ${ }^{26-29)}$ Thus, isoflavones may regulate pathological immune responses through controlling RA levels without causing side effects in the gut.

Genistin significantly suppressed the development of food allergy and was more effective than the isoflavone aglycon genistein, although genistin and genistein were almost equally effective in downregulating Cyp26b1 mRNA expression in the in vitro assay. Isoflavones are known to exert many biological functions. Masilamani et ll $^{12)}$ reported that isoflavones could suppress the maturation of DCs in the MLN and DC-mediated $\mathrm{CD}^{+} \mathrm{T}$ cell functions in vitro, thereby suppressing allergic sensitization to peanuts in a mouse model. In addition, isoflavones inhibit the degranulation of mast cells by suppressing the levels of the phosphorylated Lyn and Syk proteins and reducing the cell surface expression of $\operatorname{Fc} \varepsilon \mathrm{RI} \alpha{ }^{14)}$ We also have reported that puerarin upregulates the expression of Aldhlal in the colonic epithelial cells of food allergy mice. ${ }^{16)}$ The pres- ent study provides novel evidence that isoflavones certainly reduce Cyp26b1 expression in the intestinal mucosa, thereby suppressing allergic symptoms in food allergy mice.

Acknowledgments This study was supported by JSPS KAKENHI Grant Nos. JP16K09234 and JP19K07908 (TY) and Dannon Institute of Japan Foundation (YN).

Conflict of Interest The authors declare no conflict of interest.

Supplementary Materials The online version of this article contains supplementary materials.

\section{REFERENCES}

1) White JA, Guo YD, Baetz K, Beckett-Jones B, Bonasoro J, Hsu KE Dilworth FJ, Jones G, Petkovich M. Identification of the retinoic acid-inducible all-trans-retinoic acid 4-hydroxylase. J. Biol. Chem., 271, 29922-29927 (1996).

2) Bhattacharya N, Yuan R, Prestwood TR, Penny HL, DiMaio MA, Reticker-Flynn NE, Krois CR, Kenkel JA, Pham TD, Carmi Y, Tolentino L, Choi O, Hulett R, Wang J, Winer DA, Napoli JL, Engleman EG. Normalizing microbiota-induced retinoic acid deficiency stimulates protective $\mathrm{CD}^{+} \mathrm{T}$ cell-mediated immunity in colorectal cancer. Immunity, 45, 641-655 (2016).

3) Takeuchi H, Yokota A, Ohoka Y, Iwata M. Cyp26b1 regulates retinoic acid-dependent signals in T cells and its expression is inhibited by transforming growth factor-beta. PLoS ONE, 6, e16089 (2011).

4) Chenery A, Burrows K, Antignano F, Underhill TM, Petkovich M, Zaph C. The retinoic acid-metabolizing enzyme Cyp26b1 regulates CD4 $\mathrm{T}$ cell differentiation and function. PLOS ONE, 8, e72308 (2013).

5) Coombes JL, Siddiqui KR, Arancibia-Carcamo CV, Hall J, Sun CM, Belkaid Y, Powrie F. A functionally specialized population of mucosal $\mathrm{CD}_{103^{+}}$DCs induces Foxp ${ }^{+}$regulatory T cells via a TGFbeta and retinoic acid-dependent mechanism. J. Exp. Med., 204, 1757-1764 (2007).

6) Yamamoto T, Fujiwara K, Yoshida M, Kageyama-Yahara N, Kuramoto H, Shibahara N, Kadowaki M. Therapeutic effect of kakkonto in a mouse model of food allergy with gastrointestinal symptoms. Int. Arch. Allergy Immunol., 148, 175-185 (2009).

7) Yamamoto T, Fujiwara K, Tsubota Y, Kageyama-Yahara N, Hayashi S, Kadowaki M. Induction of regulatory $\mathrm{T}$ cells as a novel mechanism underlying the therapeutic action of kakkonto, a traditional Japanese herbal medicine, in a murine food allergy model. Int. 
Arch. Allergy Immunol., 169, 146-156 (2016).

8) Nagata Y, Yamamoto T, Hayashi M, Hayashi S, Kadowaki M. Improvement of therapeutic efficacy of oral immunotherapy in combination with regulatory $\mathrm{t}$ cell-inducer kakkonto in a murine food allergy model. PLOS ONE, 12, e0170577 (2017).

9) Reppert A, Yousef GG, Rogers RB, Lila MA. Isolation of radiolabeled isoflavones from kudzu (Pueraria lobata) root cultures. $J$. Agric. Food Chem., 56, 7860-7865 (2008).

10) Fang C, Wan X, Tan H, Jiang C. Identification of isoflavonoids in several kudzu samples by high-performance liquid chromatography coupled with electrospray ionization tandem mass spectrometry. $J$. Chromatogr. Sci., 44, 57-63 (2006).

11) Cherdshewasart W, Subtang S, Dahlan W. Major isoflavonoid contents of the phytoestrogen rich-herb Pueraria mirifica in comparison with Pueraria lobata. J. Pharm. Biomed. Anal., 43, 428-434 (2007).

12) Masilamani M, Wei J, Bhatt S, Paul M, Yakir S, Sampson HA. Soybean isoflavones regulate dendritic cell function and suppress allergic sensitization to peanut. J. Allergy Clin. Immunol., 128, 1242-1250.el (2011)

13) Park DK, Choi WS, Park HJ. Antiallergic activity of novel isoflavone methyl-glycosides from Cordyceps militaris grown on germinated soybeans in antigen-stimulated mast cells. J. Agric. Food Chem., 60, 2309-2315 (2012).

14) Yamashita S, Tsukamoto S, Kumazoe M, Kim YH, Yamada K, Tachibana H. Isoflavones suppress the expression of the FcepsilonRI high-affinity immunoglobulin E receptor independent of the estrogen receptor. J. Agric. Food Chem., 60, 8379-8385 (2012).

15) Lepri SR, Sartori D, Semprebon SC, Baranoski A, Coatti GC, Mantovani MS. Genistein affects expression of cytochrome P450 (CYP450) genes in hepatocellular carcinoma (HEPG2/C3A) cell line. Drug Metab. Lett., 12, 138-144 (2018).

16) Yamamoto $T$, Matsunami E, Komori K, Hayashi S, Kadowaki $\mathrm{M}$. The isoflavone puerarin induces Foxp ${ }^{+}$regulatory $\mathrm{T}$ cells by augmenting retinoic acid production, thereby inducing mucosal immune tolerance in a murine food allergy model. Biochem. Biophys. Res. Commun., 516, 626-631 (2019).

17) Hall JA, Cannons JL, Grainger JR, Dos Santos LM, Hand TW, Naik S, Wohlfert EA, Chou DB, Oldenhove G, Robinson M, Grigg ME, Kastenmayer R, Schwartzberg PL, Belkaid Y. Essential role for retinoic acid in the promotion of $\mathrm{CD}^{+} \mathrm{T}$ cell effector responses via retinoic acid receptor alpha. Immunity, 34, 435-447 (2011).

18) Qin W, Zhu W, Shi H, Hewett JE, Ruhlen RL, MacDonald RS,
Rottinghaus GE, Chen YC, Sauter ER. Soy isoflavones have an antiestrogenic effect and alter mammary promoter hypermethylation in healthy premenopausal women. Nutr. Cancer, 61, 238-244 (2009).

19) Stoney PN, Fragoso YD, Saeed RB, Ashton A, Goodman T, Simons C, Gomaa MS, Sementilli A, Sementilli L, Ross AW, Morgan PJ, McCaffery PJ. Expression of the retinoic acid catabolic enzyme CYP26B1 in the human brain to maintain signaling homeostasis. Brain Struct. Funct., 221, 3315-3326 (2016).

20) Takeuchi H, Yokota A, Ohoka Y, Iwata M. Cyp26b1 Regulates retinoic acid-dependent signals in T cells and its expression is inhibited by transforming growth factor- $\beta$. PLoS ONE, 6, e16089 (2011).

21) Kumari S, Raines JM, Martin JM, Rodriguez JM. Thermal stability of kudzu root (Pueraria radix) isoflavones as additives to beef patties. J. Food Sci. Technol., 52, 1578-1585 (2015).

22) Zhou $X$, Seto SW, Chang D, Kiat H, Razmovski-Naumovski V, Chan K, Bensoussan A. Synergistic effects of Chinese herbal medicine: a comprehensive review of methodology and current research. Front. Pharmacol., 7, 201 (2016).

23) Nelson $\mathrm{CH}$, Buttrick BR, Isoherranen N. Therapeutic potential of the inhibition of the retinoic acid hydroxylases CYP26A1 and CYP26B1 by xenobiotics. Curr. Top. Med. Chem., 13, 1402-1428 (2013).

24) Rigopoulos D, Larios G, Katsambas AD. The role of isotretinoin in acne therapy: why not as first-line therapy? facts and controversies. Clin. Dermatol., 28, 24-30 (2010).

25) Melling MA, Friendship CR, Shepherd TG, Drysdale TA. Expression of Ski can act as a negative feedback mechanism on retinoic acid signaling. Dev. Dyn., 242, 604-613 (2013).

26) Huynh CK, Brodie AM, Njar VC. Inhibitory effects of retinoic acid metabolism blocking agents (RAMBAs) on the growth of human prostate cancer cells and LNCaP prostate tumour xenografts in SCID mice. Br. J. Cancer, 94, 513-523 (2006).

27) Pavez Loriè E, Cools M, Borgers M, Wouters L, Shroot B, Hagforsen E, Torma H, Vahlquist A. Topical treatment with CYP26 inhibitor talarozole (R115866) dose dependently alters the expression of retinoid-regulated genes in normal human epidermis. $B r . J$. Dermatol., 160, 26-36 (2009).

28) Berth-Jones J, Todd G, Hutchinson PE, Thestrup-Pedersen K, Vanhoutte FP. Treatment of psoriasis with oral liarozole: a dose-ranging study. Br. J. Dermatol., 143, 1170-1176 (2000).

29) Thatcher JE, Isoherranen N. The role of CYP26 enzymes in retinoic acid clearance. Expert Opin. Drug Metab. Toxicol., 5, 875-886 (2009). 